\title{
Palliation of Ulcerative Breast Lesions with Radiation
}

\author{
PRASHANT VEMPATI ${ }^{1}$, MIRIAM A. KNOLL ${ }^{1}$, KAVITA DHARMARAJAN ${ }^{1}$, \\ SHERYL GREEN $^{1}$, AMY TIERSTEN ${ }^{2}$ and RICHARD L. BAKST ${ }^{1}$ \\ Departments of ${ }^{1}$ Radiation Oncology, and ${ }^{2}$ Medical Oncology, \\ Icahn School of Medicine at Mount Sinai, New York, NY, U.S.A.
}

\begin{abstract}
Background/Aim: Patients with advanced breast cancer may experience ulcerative breast lesions. Breast cancer with ulcerative lesions has been shown to severely affect a patient's quality of life $(Q o L)$. The role of palliative radiation therapy $(R T)$ in the management of ulcerative breast lesions needs to be further explored. Patients and Methods: We retrospectively reviewed the RT records for all patients who underwent palliative $R T$ for breast cancer at our urban academic medical center. A total of 13 patients were identified, and we herein report their demographics, treatment characteristics, and clinical outcomes. Results: The mean age of the patients receiving palliative $R T$ for ulcerative breast cancer was 64 years. All patients had stage $I V$ disease when they were evaluated for RT. The mean radiation dose received for palliative $R T$ was $27.54 \mathrm{~Gy}$ in 11 fractions, with a median dose of $30 \mathrm{~Gy}$ in 15 fractions. Six (46\%) patients had received prior RT to the same breast, with a median dose of 59.5 Gy in 31 fractions. Among these six patients, the average interval between initial $R T$ and ulceration was 69.5 months. The median overall survival for the whole patient cohort since ulceration was 5 months and the mean survival did not differ between patients with previous history of RT and RT-naïve patients (4.50 vs. 4.57; $p=0.95)$. Six out of the nine (69\%) patients who received 30 Gy or more reported clinical improvement, whereas none of the four patients who received less than 30 Gy reported any benefit. There were no radiation-associated toxicities reported by patients. Conclusion: These data suggest that palliative $R T(\geq 30 \mathrm{~Gy})$ is an efficacious treatment for ulcerative breast cancer with minimal toxicity. Prior $R T$
\end{abstract}

This article is freely accessible online.

Correspondence to: Richard L. Bakst, MD, Department of Radiation Oncology, Mount Sinai School of Medicine,New York, NY 10029, U.S.A. Tel: +1 2122412545, e-mail: Richard.Bakst@mountsinai.org

Key Words: Palliative radiotherapy, breast cancer, palliative care, ulcerative breast lesions. should not be a contraindication, as patients with previous history of RT have similar low toxicity rates compared to RTnaïve patients.

Initial breast cancer presentation can vary greatly. In developed countries with routine screening and awareness, breast cancer is usually detected early and presents as earlystage breast cancer. Locally advanced breast cancer (LABC), defined as primary tumor greater than $5 \mathrm{~cm}$, with or without nodal involvement, only occurs in $5 \%$ to $10 \%$ of cases in developed countries (1-7). However, these numbers are much higher in the developing world due to lack of adequate screening, awareness, and accessibility to healthcare resources $(1,3,4,7)$.

In developed countries, patients who present with LABC can be divided into two groups: Those who present without prior treatment, and those who present with persistent or recurrent $\mathrm{LABC}$ after prior treatment. Patients presenting with fungating wounds are usually plagued by chronic pain, malodorous discharges, bleeding, exudative secretions and frequent infections $(4,5,8)$ (Figure 1). These wounds can have serious physical, psychological, and social implications for patients regardless of the prognosis. As such, women diagnosed with LABC can benefit from palliative care management by an interdisciplinary team.

Within the palliative realm, there are treatments ranging from aggressive surgical intervention and intra-arterial chemotherapy to topical palliative emollients (9-13). There are very limited data regarding the effectiveness of the different treatment paradigms and far less consensus regarding the optimal treatment paradigms. Radiation therapy (RT) has been and continues to be used in patients with ulcerative breast lesions to reduce tumor burden, provide symptomatic relief, and improve quality of life (QoL). However, there are no available studies that looked at patient characteristics and clinical outcomes of the palliative RT of ulcerative breast lesions.

We look at our own experience to assess patient demographics, treatment characteristics, and clinical outcomes. The purpose of this study was to offer recommendations for 
Table I. Baseline characteristics of the cohort.

\begin{tabular}{lcc}
\hline & Prior RT y (n=6) & RT-naïve (n=7) \\
\hline Mean age, years & 64 & 64 \\
Median DFI (range) from initial treatment to ulcerative breast lesion, months & $69.5(34-167)$ & Not applicable \\
Average no. of previous chemotherapy trials & 3.67 & 3.17 \\
Average no. of previous hormonal therapies & 1.29 & 1.33 \\
Average dose of previous RT, Gy & 58 & Not applicable \\
Median dose of previous RT, Gy & 59.50 & Not applicable \\
Tumor histology & & 0.67 \\
ER+, PR+, HER2- & 0 & 4 \\
ER+, PR-, HER2- & 2 & 1 \\
ER-, PR-, HER2- & 4 & 2 \\
\hline
\end{tabular}

DFI: Disease-free interval; RT: radiotherapy; ER: estrogen receptor; PR: progesterone receptor; HER2: human epidermal growth factor receptor 2.

palliative treatment of ulcerative breast lesions based on our past experience.

\section{Patients and Methods}

This study was approved by the Institutional Review Board at our institution. We performed a retrospective review of the electronic medical records of patients treated from 2006-2015 at our hospital. A total of 13 patients underwent palliative radiation to ulcerative breast lesions with available medical records. Patient demographics, previous and current treatment characteristics, and clinical outcomes were obtained from the patients' electronic medical records. Treatment benefit was assessed by patient reported or physician observed benefit explicitly noted on the patients' charts. Outcomes included a reduction in ulceration, pain, or bleeding, Acute and late toxicities were recorded using Radiation Therapy Oncology Group/European Organisation for Research and Treatment of Cancer (RTOG/EORTC) toxicity grading scale.

Statistical analysis. Statistical analysis was performed using SPSS version 23 (IBM Corp., Armonk, NY, USA). A two sample $t$-test was used to calculate differences in patient characteristics and treatment regimens. An N-1 two proportion test was used to compare patient-experienced benefit between the groups.

\section{Results}

Patient characteristics. A total of 13 patients were identified who received palliative doses of radiation to ulcerative breast lesions. The mean age of the patients receiving palliative RT for ulcerative breast cancer was 64 years (range $=34$ 95 years). All of the patients were female and there were no significant differences in patient characteristics (Table I). All of the patients had received previous treatment for breast cancer with chemotherapy (no prior surgeries) and six out of the 13 patients had received prior RT to the same breast.

Treatment. The median dose of radiation for the whole cohort was $30 \mathrm{~Gy}$; the median dose of radiation for patients with previous history of RT was 30 Gy whereas that for patients without previous history of RT was $32.5 \mathrm{~Gy}$. The median fractionation for the whole cohort was 13 fractions; the median fractionation for patients with prior history of radiation was 14 fractions, whereas that for patients without prior radiation was 10. The radiation dosage did not differ significantly between patients with and patients without previous RT.

Outcomes. The mean survival for all patients who received palliative RT was 4.5 months. The mean survival for patients with prior RT was 4.6 months, whereas that for patients without prior RT was 4.5 months $(p=0.95)$. Three out of six patients from the group with prior RT experienced benefit from the palliative treatment and three out of seven patients from the RT-naive group experienced benefit from the palliative treatment $(p=0.81)$. In regards to dose, six out of nine patients from the group receiving RT of $30 \mathrm{~Gy}$ or more observed benefit of their treatment, whereas none of the patients in the group receiving less than 30 Gy RT observed any benefit from the treatment $(p=0.033)$ (Table II). There were no RT-associated toxicities reported by patients.

Sample case. A 55-year-old female with an eight-year history of estrogen receptor-, progesterone receptor- and HER2/neu receptor-positive $\left(\mathrm{ER}^{+} \mathrm{PR}{ }^{+} \mathrm{HER} 2 / \mathrm{neu}^{+}\right)$-positive, poorly differentiated invasive ductal carcinoma presented on $04 / 2015$ with a 1 -month history of an ulcerative lesion of the right breast. The patient was initially diagnosed with metastatic (bone, lung) breast cancer at another hospital in December 2008 and had been receiving treatment since. The patient had progression of the disease despite being on multiple chemotherapy trials including, Herceptin, XGEVA (denosumab), Perjeta (pertuzumab), tamoxifen, everolimus, KADCYLA (ado-trastuzumab emtansine).

At initial diagnosis, the patient had received 66 Gy in 33 fractions of external beam radiation to the right breast and 

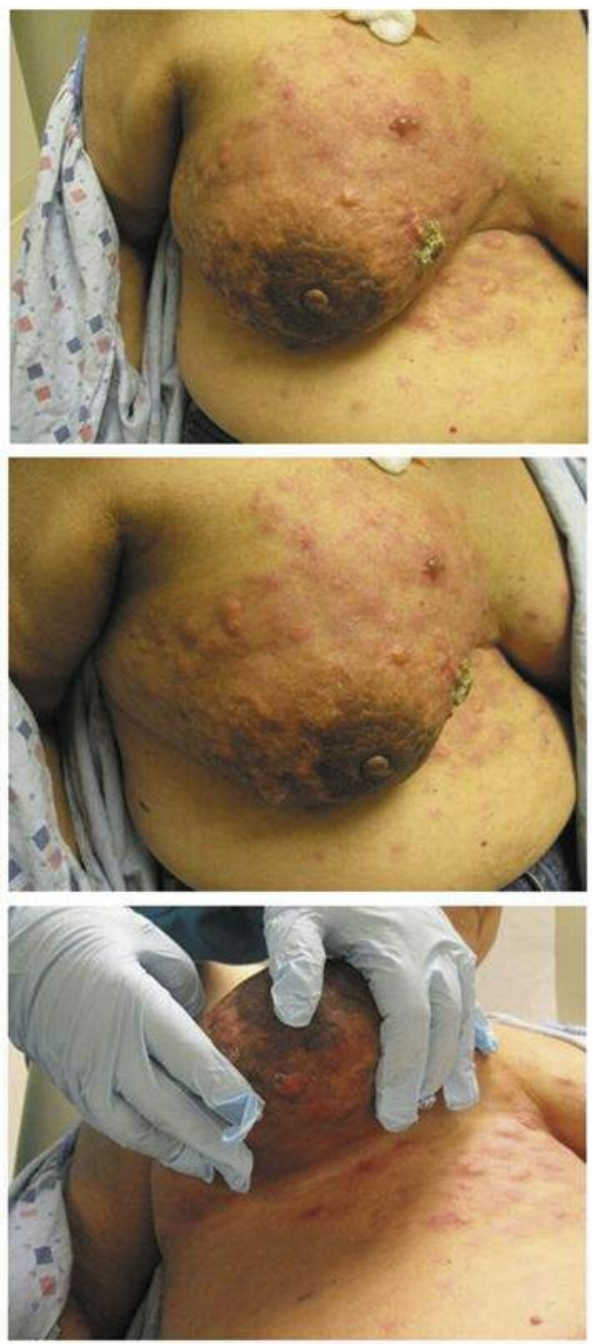

Figure 1. Example of an ulcerative breast lesion of the right breast with tender erythematous skin lesions and multiple nodules with bullae and crusting.

$30 \mathrm{~Gy}$ in 15 fractions to the lumbosacral region of the spine at another hospital. Although she responded initially to the radiation, she eventually had recurrence of disease in the right breast region.

Three years later, the patient was found to have an ulcerative lesion that was causing her significant distress and as such, she was referred to Radiation Oncology for continued management. The patient complained of pain and constant "oozing". On examination, the patient was found to have a mass of the right breast with a tender, erythematous, $2 \times 1 \mathrm{~cm}$ skin lesion on the upper inner quadrant. Positronemission tomography/computed tomography (PET/CT) showed progression of disease on her current chemotherapy treatment of Herceptin and Kadcyla (Figure 2A). Based on
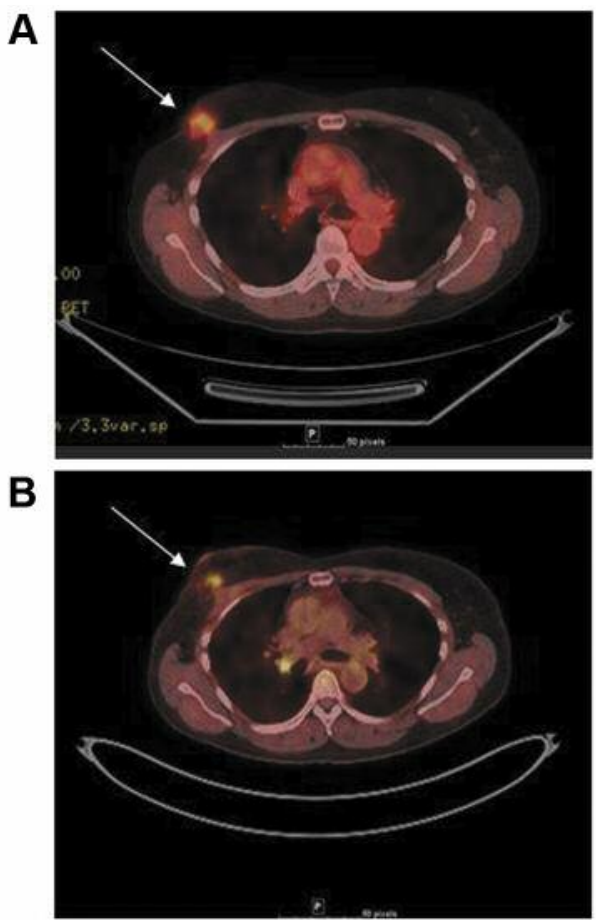

Figure 2. A: Pre-palliative radiation therapy positron-emission tomography/computed tomography (PET/CT) showing increased uptake in the right breast region in an axial plane, standardized uptake value (SUV)max=5.0. B: Three-month post-palliative radiation therapy PET/CT image showing decreased uptake in the right breast region with regression from the skin surface in an axial plane, with improvement of SUVmax (3.2).

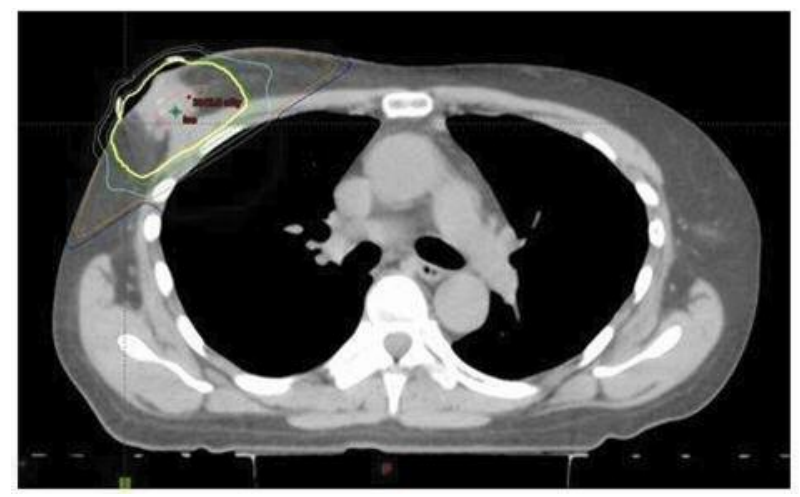

Figure 3. 3D-Conformal radiation therapy of ulcerative breast lesion with dose distribution depicted in coronal plane. Yellow: 30 Gy isodose line; orange: 15 Gy isodose line.

the patient's presentation and our previous experience with ulcerative breast lesions, we decided to proceed with 30-Gy external beam RT in 15 fractions. Subsequently, the patient underwent simulation for RT and treatment with 3D 
Table II. Clinical benefit achieved from radiation (RT) as a function of dose.

\begin{tabular}{lccc}
\hline & $<30$ Gy RT $(\mathrm{n}=4)$ & $\geq 30$ Gy RT $(\mathrm{n}=9)$ & $p$-Value \\
\hline Mean no. of fractions of palliative treatment & 5 & 13 & 0.000000824 \\
Patient benefit from RT & 0 & 6 & 0.033 \\
\hline
\end{tabular}

conformal RT technique to treat the ulcerative breast lesion (Figure 3). At 1 month post-RT visit, the patient reported significant resolution of pain and "oozing". At 3 months post-radiotherapy, PET/CT showed significant resolution of uptake in the right breast and decreased involvement of the skin and superficial subcutaneous layers of right breast (Figure 2B).

\section{Discussion}

Although screening mammography and advances in breast cancer treatment have markedly reduced the incidence of LABC (3), there is still a significant number of patients presenting either with neglected locally advanced disease or with metastatic disease with locally aggressive primaries unsuitable for surgery. Ulcerative breast lesions characterized by a fungating mass infiltrating the surrounding breast tissue can be physically and psychologically damaging for the patient. Patients with ulcerative breast lesions usually present with large open wounds, chronic pain, malodor, frequent infections and other physical and psychological symptoms.

Thirteen women presented to our Department with ulcerative breast lesions over the course of a 9-year period. All these women were evaluated by our multidisciplinary team, which included surgeons, medical oncologists, and radiation oncologists, among other professionals. All patients had received previous chemotherapy and six patients had received prior radiation to the same breast. Prior treatment, specifically prior RT to the same area of disease, might be viewed cautiously as re-irradiation can have significant side-effects, including tissue necrosis.

In our patient population, prior RT to the same breast did not cause any complications for palliative treatment. This may be partly due to the long interval between the initial RT and the presentation of ulcerative breast lesion and subsequent palliative RT for our patients (mean=69.5 months). In addition, the overall survival following ulcerative lesions can be limited, as in our series the mean survival was 4.54 months following RT. Therefore, long-term sequelae are also less of a concern in patients with limited survival. As we did not observe any short-term or long-term toxicities in our study, we conclude that prior radiation should not be a contraindication for palliative RT of ulcerative breast lesions.
In terms of the RT dose itself, patients who received greater than 30 Gy of RT (six out of nine patients) experienced a benefit of RT, whereas patients who received less than $30 \mathrm{~Gy}$ of RT did not experience any benefit from RT. Benefit of RT included pain resolution, bleeding cessation, wound improvement, and improved appearance of the site. While RT dose is important, other treatment considerations, such as fractionation, treatment volume, and overall treatment time, can also play a significant role in palliative treatments. Based on our small series, we recommend $>30$ Gy in standard fractionation to the ulcerative breast lesion or equivalent biologically effective dose (BED) if using hypofractionation, regardless of previous RT treatment. Hypofractionation is encouraged, especially in the setting of no prior RT, a limited life expectancy or the need to resume systemic therapy quickly.

Limitations of this study include its small sample size and retrospective nature. Future studies will improve upon these limitations by including a larger sample size and creating a standardized method to assess improvement of ulcerative breast lesions due to palliative RT.

\section{Conflicts of Interest}

The Authors have no actual or potential conflicts of interest. Amy Tiersten, MD serves on the speakers bureau for Celgene, Amgen, and Pfizer; however, this does not represent a conflict of interest as it relates to this article.

\section{References}

1 Bottomley A, Therasse P, Piccart M, Efficace F, Coens C, Gotay C, Welnicka-Jaskiewicz M, Mauriac L, Dyczka J, Cufer T, Lichinitser MR, Schornagel JH, Bonnefoi H, Shepherd L, European Organisation for Research and Treatment of Cancer Breast Cancer Group, National Cancer Institute of Canada, Swiss Group for Clinical Cancer Research. Health-related quality of life in survivors of locally advanced breast cancer: an international randomised controlled phase III trial. Lancet Oncol 6(5): 287-294, 2005.

2 Bogusevicius A, Cepuliene D, Sepetauskiene E. The integrated evaluation of the results of oncoplastic surgery for locally advanced breast cancer. Breast J 20(1): 53-60, 2014.

3 Coebergh J, Janssen-Heijnen M, Louwman W, Voogd A. Cancer incidence and survival in the south of the Netherlands, 19551999 and incidence in the north of Belgium, 1996-1998. Eindhoven: Comprehensive Cancer Centre South (IKZ), 2001. 
4 Jarvis V: The range and role of palliative interventions for locally advanced breast cancer. Curr Opin Support Palliat Care 8(1): 70-76, 2014.

5 Lee MC and Newman LA: Management of patients with locally advanced breast cancer. Surg Clin North Am 87(2): 379-398, 2007

6 Sood A, Daniali LN, Rezzadeh KS, Lee ES and Keith J: Management and Reconstruction in the Breast Cancer Patient With a Fungating T4b Tumor. Eplasty 15: 39, 2015.

7 Wieland AWJ, Louwman MWJ, Voogd AC, van Beek MWPM, Vreugdenhil G and Roumen RMH: Determinants of prognosis in breast cancer patients with tumor involvement of the skin (pT4b). Breast J 10(2): 123-128, 2004.

8 Lund-Nielsen B, Müller K and Adamsen L: Malignant wounds in women with breast cancer: feminine and sexual perspectives. J Clin Nurs 14(1): 56-64, 2005.

9 Alexander S: Malignant fungating wounds: epidemiology, aetiology, presentation and assessment. J Wound Care 18(7): 273-280, 2009.
10 da Costa Santos CM, de Mattos Pimenta CA and Nobre MRC: A systematic review of topical treatments to control the odor of malignant fungating wounds. J Pain Symptom Manage 39(6): 1065-1076, 2010.

11 Bufill JA, Grace WR and Neff R: Intra-arterial chemotherapy for palliation of fungating breast cancer. A case report and review of the literature. Am J Clin Oncol 17(2): 118-124, 1994.

12 Sanders R and Goodacre TE: When radiotherapy offers no more: the surgical management of advanced breast malignancy. Ann R Coll Surg Engl 71(6): 349-353, 1989.

13 Woo KY and Sibbald RG: Local wound care for malignant and palliative wounds. Adv Skin Wound Care 23(9): 417-428-430, 2010 .

Received June 22, 2016

Revised July 28, 2016

Accepted July 29, 2016 